MATHEMATICS OF COMPUTATION

Volume 70, Number 235, Pages 1113-1130

S 0025-5718(00)01240-0

Article electronically published on March 1, 2000

\title{
SPHERICAL MARCINKIEWICZ-ZYGMUND INEQUALITIES AND POSITIVE QUADRATURE
}

\author{
H. N. MHASKAR, F. J. NARCOWICH, AND J. D. WARD
}

\begin{abstract}
Geodetic and meteorological data, collected via satellites for example, are genuinely scattered and not confined to any special set of points. Even so, known quadrature formulas used in numerically computing integrals involving such data have had restrictions either on the sites (points) used or, more significantly, on the number of sites required. Here, for the unit sphere embedded in $\mathbb{R}^{q}$, we obtain quadrature formulas that are exact for spherical harmonics of a fixed order, have nonnegative weights, and are based on function values at scattered sites. To be exact, these formulas require only a number of sites comparable to the dimension of the space. As a part of the proof, we derive $L^{1}$-Marcinkiewicz-Zygmund inequalities for such sites.
\end{abstract}

\section{INTRODUCTION}

In many practical applications, one needs to evaluate certain integrals on a sphere embedded in a Euclidean space. Often, these integrals cannot be computed in closed form, and a quadrature (numerical integration, cubature) formula is necessary. For example, given that the gravitational potential is assumed known on the earth's surface, its value at other levels is given by a convolution integral ([6] p. 9]). Numerical estimates of its actual values were given in [6] using low accuracy quadrature rules. Certain initial value problems involving the Gauss-Weierstrass (heat) kernels have solutions expressed as convolution integrals (see 7, p. 6]). Again, application of high accuracy quadrature rules will give accurate numerical solutions. More generally, whenever Galerkin techniques are used to solve differential equations numerically, high accuracy quadrature formulas are of critical importance.

Let $q \geq 1$ be an integer, and $\mathbb{S}^{q}$ denote the (surface of the) unit sphere embedded in the Euclidean space $\mathbb{R}^{q}$. In this paper, we obtain quadrature formulas that use function values at scattered points (sites) on $\mathbb{S}^{q}$. These formulas are exact for spherical harmonics of degree less than or equal to a given integer $n$, and have nonnegative weights that can be computed by known methods from linear or quadratic

Received by the editor January 26, 1999 and, in revised form, August 25, 1999.

2000 Mathematics Subject Classification. Primary 65D32; Secondary 41A17, 42C10.

Key words and phrases. Marcinkiewicz-Zygmund inequalities, quadrature, scattered-data on spheres.

Research of the authors was sponsored by the Air Force Office of Scientific Research, Air Force Materiel Command, USAF, under grant numbers F49620-97-1-0211 and F49620-98-1-0204. The U.S. Government is authorized to reproduce and distribute reprints for governmental purposes notwithstanding any copyright notation thereon. The views and conclusions contained herein are those of the authors and should not be interpreted as necessarily representing the official policies or endorsements, either expressed or implied, of the Air Force Office of Scientific Research or the U.S. Government.

(C) 2000 American Mathematical Society 
programming. The number of sites required is comparable to the dimension of the spherical harmonics for which the formulas are exact.

In the case of "equal-angle" sites, Driscoll and Healy [2] and Potts, Steidl, and Tasche [17] obtained quadrature formulas on $\mathbb{S}^{q}$ that have the other properties mentioned above. More recently, Petrushev [16] found quadrature formulas that use specific sites, which are not "equal-angle", that collect around the poles of $S^{q}$. Jetter, Stöckler, and Ward [11] obtained quadrature formulas for scattered sites; these formulas used weights that were real, but possibly negative.

The quadrature formulas given in this paper are based on new MarcinkiewiczZygmund inequalities for $\mathbb{S}^{q}$, which are derived in Section 3 . These inequalities provide isomorphisms between the space of spherical harmonics having degree at most $n$ and the space comprising the restrictions of these spherical harmonics to finitely many scattered sites; the former space is endowed with the $L^{p}$ norm, and the latter, with a weighted $\ell^{p}$ norm. The results derived in this paper are apparently new even in the case of the circle.

In addition to being of central importance in the development of quadrature formulas, the Marcinkiewicz-Zygmund inequalities play a role in establishing uniform boundedness of discretized operators used to approximately reconstruct functions from data sampled at scattered sites. We will discuss these operators and the related reconstruction methods in a follow-up paper [13].

The outline of this paper is as follows. In the next section, after summarizing certain central facts about spherical harmonics and establishing notation, we discuss delayed-mean operators, which were introduced by Stein [18. These kernels, together with an adaptation of the Mastroianni-Totik theory of doubling weights, discussed in subsection 3.1 and decompositions of $\mathbb{S}^{q}$ related to scattered sites, developed in subsection 3.2, are the ingredients we use in subsection 3.3 to prove the estimate that is central to obtaining $L^{p}$ versions of Marcinkiewicz-Zygmund (MZ) inequalities for $\mathbb{S}^{q}$. In Section 4, we use the MZ inequalities and the estimate from subsection 3.3 to obtain scattered site quadrature formulas for $\mathbb{S}^{q}$. In that section, we also discuss ways of computing the weights.

\section{Delayed means}

2.1. Spherical harmonics. Let $q \geq 1$ be an integer which will be fixed throughout the rest of this paper, and let $\mathbb{S}^{q}$ be the (surface of the) unit sphere in the Euclidean space $\mathbb{R}^{q+1}$, with $d \mu_{q}$ being its usual volume element. We note that the volume element is invariant under arbitrary coordinate changes. The volume of $\mathbb{S}^{q}$ is

$$
\omega_{q}:=\int_{\mathbb{S} q} d \mu_{q}=\frac{2 \pi^{(q+1) / 2}}{\Gamma((q+1) / 2)} .
$$

Corresponding to $d \mu_{q}$, we have the inner product and $L^{p}\left(\mathbb{S}^{q}\right)$ norms,

$$
\begin{aligned}
\langle f, g\rangle_{\mathbb{S} q} & :=\int_{\mathbb{S}^{q}} f(\mathbf{x}) \overline{g(\mathbf{x})} d \mu_{q}(\mathbf{x}), \\
\|f\|_{\mathbb{S}^{q}, p} & := \begin{cases}\left\{\int_{\mathbb{S}^{q}}|f(\mathbf{x})|^{p} d \mu_{q}(\mathbf{x})\right\}^{1 / p} & \text { if } 1 \leq p<\infty \\
\underset{\mathbf{t} \in \mathbb{S}}{\operatorname{ess} \sup }|f(\mathbf{t})| & \text { if } p=\infty .\end{cases}
\end{aligned}
$$

The class of all measurable functions $f: \mathbb{S}^{q} \rightarrow \mathbb{C}$ for which $\|f\|_{\mathbb{S} q, p}<\infty$ will be denoted by $L^{p}\left(\mathbb{S}^{q}\right)$, with the usual understanding that functions that are equal 
almost everywhere are considered equal as elements of $L^{p}\left(\mathbb{S}^{q}\right)$. All continuous complex valued functions on $\mathbb{S}^{q}$ will be denoted by $C\left(\mathbb{S}^{q}\right)$.

For integer $\ell \geq 0$, the restriction to $\mathbb{S}^{q}$ of a homogeneous harmonic polynomial of degree $\ell$ is called a spherical harmonic of degree $\ell$. Most of the following information is based on [15] and [19, §IV.2], although we use a different notation. The class of all spherical harmonics of degree $\ell$ will be denoted by $\mathbf{H}_{\ell}^{q}$, and the class of all spherical harmonics of degree $\ell \leq n$ will be denoted by $\Pi_{n}^{q}$. Of course, $\Pi_{n}^{q}=\bigoplus_{\ell=0}^{n} \mathbf{H}_{\ell}^{q}$, and it comprises the restriction to $\mathbb{S}^{q}$ of all algebraic polynomials in $q+1$ variables of total degree not exceeding $n$. The dimension of $\mathbf{H}_{\ell}^{q}$ is given by

$$
d_{\ell}^{q}:=\operatorname{dim} \mathbf{H}_{\ell}^{q}=\left\{\begin{array}{cc}
\frac{2 \ell+q-1}{\ell+q-1}\left(\begin{array}{c}
\ell+q-1 \\
\ell
\end{array}\right) & \text { if } \ell \geq 1, \\
1 & \text { if } \ell=0,
\end{array}\right.
$$

and that of $\Pi_{n}^{q}$ is $\sum_{\ell=0}^{n} d_{\ell}^{q}$.

The spherical harmonics have an intrinsic characterization as the eigenfunctions of the Laplace-Beltrami operator, $\Delta_{\mathbb{S} q}$. The operator $\Delta_{\mathbb{S} q}$ is an elliptic, (unbounded) self-adjoint operator on $L^{2}\left(\mathbb{S}^{q}\right)$, is invariant under arbitrary coordinate changes, and its spectrum comprises distinct eigenvalues $\lambda_{\ell}:=\ell(\ell+q-1), \ell=0,1, \ldots$, each having finite multiplicity, $d_{\ell}^{q}$. The space $\mathbf{H}_{\ell}^{q}$ can be characterized intrinsically as the eigenspace corresponding to $\lambda_{\ell}$.

Since the $\lambda_{\ell}$ 's are distinct and the operator is self-adjoint, the spaces $\mathbf{H}_{\ell}^{q}$ 's are mutually orthogonal relative to (2.2). Also, $L^{2}\left(\mathbb{S}^{q}\right)=$ closure $\left\{\bigoplus_{\ell} \mathbf{H}_{\ell}^{q}\right\}$. Hence, if we choose an orthonormal basis $\left\{Y_{\ell, k}: k=1, \ldots, d_{\ell}^{q}\right\}$ for each $\mathbf{H}_{\ell}^{q}$, then the set $\left\{Y_{\ell, k}: \ell=0,1, \ldots\right.$, and $\left.k=1, \cdots, d_{\ell}^{q}\right\}$ is an orthonormal basis for $L^{2}\left(\mathbb{S}^{q}\right)$. Finally, one has the well-known addition formula [15]

$$
\sum_{k=1}^{d_{\ell}^{q}} Y_{\ell, k}(\mathbf{x}) \overline{Y_{\ell, k}(\mathbf{y})}=\frac{d_{\ell}^{q}}{\omega_{q}} \mathcal{P}_{\ell}(q+1 ; \mathbf{x} \cdot \mathbf{y}), \quad \ell=0,1, \ldots,
$$

where $\mathcal{P}_{\ell}(q+1 ; x)$ is the degree- $\ell$ Legendre polynomial in $q+1$-dimensions. (We note that Müller's $\omega_{q}$ and $N(q, \ell)$ are the same as our $\omega_{q+1}$ and $d_{\ell}^{q+1}$.)

The Legendre polynomials are normalized so that $\mathcal{P}_{\ell}(q+1 ; 1)=1$, and satisfy the orthogonality relations [15, Lemma 10]

$$
\int_{-1}^{1} \mathcal{P}_{\ell}(q+1 ; x) \mathcal{P}_{k}(q+1 ; x)\left(1-x^{2}\right)^{\frac{q}{2}-1} d x=\frac{\omega_{q}}{\omega_{q-1} d_{\ell}^{q}} \delta_{\ell, k} .
$$

They are related to the ultraspherical (Gegenbauer) polynomials $P_{\ell}^{\left(\frac{q-1}{2}\right)}$ (cf. [20], [15, p. 33]), and the Jacobi polynomials, $P_{\ell}^{(\alpha, \beta)}$, with $\alpha=\beta=\frac{q}{2}-1$, via

$$
\begin{aligned}
P_{\ell}^{\left(\frac{q-1}{2}\right)}(x) & =\left(\begin{array}{c}
\ell+q-2 \\
\ell
\end{array}\right) \mathcal{P}_{\ell}(q+1 ; x) \quad(q \geq 2), \\
P_{\ell}^{\left(\frac{q}{2}-1, \frac{q}{2}-1\right)}(x) & =\left(\begin{array}{c}
\ell+\frac{q}{2}-1 \\
\ell
\end{array}\right) \mathcal{P}_{\ell}(q+1 ; x) .
\end{aligned}
$$

When $q=1$, the Legendre polynomials $\mathcal{P}_{\ell}(2 ; x)$ coincide with the Chebyshev polynomials $T_{\ell}(x)$; the ultraspherical polynomials $P_{\ell}^{(0)}(x)=(2 / \ell) T_{\ell}(x)$, if $\ell \geq 1$. For $\ell=0, P_{0}^{(0)}(x)=1$.

In addition to the inner product and norms defined above on $\mathbb{S}^{q}$, we will need the following related inner product and norms for $[-1,1]$, with weight function 
$w_{q}(x):=\left(1-x^{2}\right)^{\frac{q}{2}-1}:$

$$
\begin{aligned}
\langle f, g\rangle_{w_{q}} & :=\int_{-1}^{1} f(x) \overline{g(x)} w_{q}(x) d x, w_{q}(x):=\left(1-x^{2}\right)^{\frac{q}{2}-1}, \\
\|f\|_{w_{q}, p} & := \begin{cases}\left\{\int_{-1}^{1}|f(x)|^{p} w_{q}(x) d x\right\}^{1 / p} & \text { if } 1 \leq p<\infty, \\
\operatorname{ess~sup}_{x \in[-1,1]}|f(x)| & \text { if } p=\infty .\end{cases}
\end{aligned}
$$

Finally, we note that for any $\phi \in L_{w_{q}}^{1}[-1,1]$, and $\mathbf{y} \in \mathbb{S}^{q}$, we have

$$
\int_{\mathbb{S} q} \phi(\mathbf{x} \cdot \mathbf{y}) d \mu_{q}(\mathbf{x})=\omega_{q-1} \int_{-1}^{1} \phi(x) w_{q}(x) d x .
$$

2.2. Cesàro means and delayed means. The addition formula allows us to express a variety of projection operators directly in terms of simple kernels. For example, the projection of $f \in L^{2}\left(\mathbb{S}^{q}\right)$ onto $\mathbf{H}_{\ell}^{q}$ is just

$$
\mathrm{P}_{\ell} f(\mathbf{x}):=\operatorname{Proj}_{\mathbf{H}_{\ell}^{q}} f(\mathbf{x})=\frac{d_{\ell}^{q}}{\omega_{q}} \int_{\mathbb{S}^{q}} \mathcal{P}_{\ell}(q+1 ; \mathbf{x} \cdot \mathbf{y}) f(\mathbf{y}) d \mu_{q}(\mathbf{y})
$$

For the sequence $\left\{\mathrm{P}_{\ell}\right\}_{\ell=0}^{n}$ of these mutually orthogonal projections, we can form the order- $k$ Cesàro means in the usual way,

$$
\Sigma_{n}^{(k)}:=\left(\begin{array}{c}
n+k \\
n
\end{array}\right)^{-1} \sum_{\ell=0}^{n}\left(\begin{array}{c}
n-\ell+k \\
n-\ell
\end{array}\right) \mathrm{P}_{\ell} .
$$

The resulting Cesàro operators have simple representations in terms of kernels. One need only replace the projections above by their kernels given in (2.12), the result being

$$
\sigma_{n}^{(k)}(\mathbf{x} \cdot \mathbf{y})=\left(\begin{array}{c}
n+k \\
n
\end{array}\right)^{-1} \sum_{\ell=0}^{n}\left(\begin{array}{c}
n-\ell+k \\
n-\ell
\end{array}\right) \frac{d_{\ell}^{q}}{\omega_{q}} \mathcal{P}_{\ell}(q+1 ; \mathbf{x} \cdot \mathbf{y})
$$

The kernel $\sigma_{n}^{(k)}$ is a univariate polynomial having degree $n$. Concerning the $L^{1}\left(w_{q}\right)$ norms of these polynomials, we have the following.

Proposition 2.1. The sequence of $L^{1}$ norms $\left\|\sigma_{n}^{(k)}\right\|_{w_{q}, 1}$ is uniformly bounded if and only if $k>\frac{q-1}{2}$.

Proof. From (2.6) and (2.10), we have $\left\|\mathcal{P}_{\ell}(q+1 ; \cdot)\right\|_{w_{q}, 2}^{2}=\frac{\omega_{q}}{\omega_{q-1} d_{\ell}^{q}}$. Since $\mathcal{P}_{\ell}(q+1 ; 1)=1$, we also have that

$$
\frac{d_{\ell}^{q}}{\omega_{q}} \mathcal{P}_{\ell}(q+1 ; x)=\omega_{q-1}^{-1}\left\|\mathcal{P}_{\ell}(q+1 ; \cdot)\right\|_{w_{q}, 2}^{-2} \mathcal{P}_{\ell}(q+1 ; 1) \mathcal{P}_{\ell}(q+1 ; x) .
$$

Because the expression on the right above is homogeneous in $\mathcal{P}_{\ell}(q+1 ; \cdot)$, we will not alter it if we replace $\mathcal{P}_{\ell}$ by the Jacobi polynomial $P_{\ell}^{\left(\frac{q}{2}-1, \frac{q}{2}-1\right)}$. Also, following 20, $\S 4.3]$, let $h^{\left(\frac{q}{2}-1, \frac{q}{2}-1\right)}=\left\|P_{\ell}^{\left(\frac{q}{2}-1, \frac{q}{2}-1\right)}\right\|_{w_{q}, 2}^{2}$. Making all of these replacements in (2.14), we obtain

$$
\sigma_{n}^{(k)}(x)=\omega_{q-1}^{-1}\left(\begin{array}{c}
n+k \\
n
\end{array}\right)^{-1} \sum_{\ell=0}^{n}\left(\begin{array}{c}
n-\ell+k \\
n-\ell
\end{array}\right)\left\{h^{(\alpha, \beta)}\right\}^{-1} P_{\ell}^{(\alpha, \beta)}(1) P_{\ell}^{(\alpha, \beta)}(x),
$$


where $\alpha=\beta=\frac{q}{2}-1$. From [20, $\left.\S 9.41\right]$, we see that the sequence $\left\|\sigma_{n}^{(k)}\right\|_{w_{q}, 1}$ is bounded if and only if $k>\alpha+\frac{1}{2}=\frac{q-1}{2}$.

It immediately follows from (2.15) and formula (2.11) that for every $g \in C\left(\mathbb{S}^{q}\right)$,

$$
\left\|\Sigma_{n}^{(k)}(g)\right\|_{\mathbb{S} q, \infty} \leq \omega_{q-1}\left\|\sigma_{n}^{(k)}\right\|_{w_{q}, 1}\|g\|_{\mathbb{S}, \infty} .
$$

Along with Proposition 2.1 this yields

Corollary 2.1. If $k>\frac{q-1}{2}$, then there is a constant $C$ such that the linear operators $\Sigma_{n}^{(k)}: L^{\infty}\left(\mathbb{S}^{q}\right) \rightarrow C\left(\mathbb{S}^{q}\right)$ satisfy $\left\|\Sigma_{n}^{(k)}\right\| \leq C$ for all $n$.

The Cesàro operators are needed to construct the delayed-mean operators introduced by Stein [18. These operators reduce to the identity on $\Pi_{n}^{q}$ and have other desirable properties. A simple example is

$$
T_{n}^{(1)}:=2 \Sigma_{2 n-1}^{(1)}-\Sigma_{n-1}^{(1)}=\sum_{\ell=0}^{n} \mathrm{P}_{\ell}+\sum_{\ell=n+1}^{2 n-1} \frac{2 n-\ell}{n} \mathrm{P}_{\ell}, n \geq 1 .
$$

The first sum on the right is the orthogonal projection onto $\Pi_{n}^{q}$ and the second maps $\Pi_{n}^{q}$ to 0 . If $q$ is 1 or 2 , then by Corollary 2.1 the sequence of operators $T_{n}^{(1)}: L^{\infty}\left(\mathbb{S}^{q}\right) \rightarrow C\left(\mathbb{S}^{q}\right)$ satisfies $\left\|T_{n}^{(1)}\right\| \leq 3 C$ for all $n \geq 1$. We remark that if $q=3$, then we need $k>(3-1) / 2=1$. In general, we denote the smallest integer $k$ for which $k>\frac{q-1}{2}$ by

$$
k_{q}:=\left\lfloor\frac{q-1}{2}\right\rfloor+1=\left\{\begin{array}{cl}
\frac{q+1}{2} & \text { if } q \text { is odd } \\
\frac{q}{2} & \text { if } q \text { is even. }
\end{array}\right.
$$

The general form of a delayed-mean operator is this:

$$
T_{n}^{(k)}:=\sum_{j=1}^{k+1} a_{j}^{k, n} \Sigma_{j n-1}^{(k)}, \quad n \geq 1 .
$$

Theorem 2.1 ([18, Theorem 1]). Let $k$ be a fixed nonnegative integer. There is a positive integer $N_{q}$ such that for $n \geq N_{q}$, one can find coefficients $a_{j}^{k, n} \in \mathbb{R}$, $j=1, \ldots, k+1$ such that $T_{n}^{(k)}: L^{\infty}\left(\mathbb{S}^{q}\right) \rightarrow C\left(\mathbb{S}^{q}\right)$ in (2.17) satisfies

$$
T_{n}^{(k)}=\sum_{\ell=0}^{n} \mathrm{P}_{\ell}+\sum_{\ell=n+1}^{(k+1) n-1} b_{\ell}^{k, n} \mathrm{P}_{\ell},
$$

where the $b_{\ell}^{k, n}$ are linear combinations of the $a_{j}^{k, n}$ 's. Moreover, the coefficients $a_{j}^{k, n}$ satisfy $\left|a_{j}^{k, n}\right| \leq A$, where $A$ is independent of $n$ and $j$. Finally, if $k \geq k_{q}$, then the norms $\left\|T_{n}^{(k)}\right\|$ are uniformly bounded in $n$.

Proof. See [18]. Only the last statement requires comment. For $k \geq k_{q}>\frac{q-1}{2}$, the Cesàro operators in (2.17) are uniformly bounded. Since the coefficients are also uniformly bounded, the norms of $T_{n}^{(k)}$ are too.

The delayed-mean operators $T_{n}^{(k)}$ inherit a kernel representation from the Cesàro operators; namely,

$$
\tau_{n}^{(k)}(\mathbf{x} \cdot \mathbf{y})=\sum_{j=1}^{k+1} a_{j}^{k, n} \sigma_{j n-1}^{(k)}(\mathbf{x} \cdot \mathbf{y})
$$


Concerning these, we obtain the following result by combining the previous results in this section.

Corollary 2.2. Let $k \geq k_{q}>\frac{q-1}{2}$ be a fixed integer. If $n \geq N_{q}$, then $\tau_{n}^{(k)}$ in (2.19) is a univariate polynomial of degree $(k+1) n-1$, the norms $\left\|\tau_{n}^{(k)}\right\|_{w_{q}, 1}$ are uniformly bounded in $n$, and $\tau_{n}^{(k)}$ is a "reproducing kernel" on $\Pi_{n}^{q}$.

From the kernel representation for $T_{n}^{(k)}$, and Theorem 1 of [14], we have the following stronger version of Theorem 2.1

Corollary 2.3. Let $1 \leq p \leq \infty$. With the notation as in Theorem 2.1, the operator norms of $T_{n}^{(k)}: L^{p}\left(\mathbb{S}^{q}\right) \rightarrow L^{p}\left(\mathbb{S}^{q}\right)$ are uniformly bounded for $k \geq k_{q}$; i.e., for every $f \in L^{p}\left(\mathbb{S}^{q}\right)$ and $k \geq k_{q}$, we have

$$
\left\|T_{n}^{(k)} f\right\|_{\mathbb{S}^{q}, p} \leq c\|f\|_{\mathbb{S}^{q}, p} .
$$

\section{MarcinkieWicZ-ZYGmund INEQUALITIES For $\mathbb{S}^{q}$}

The main objective of this section is to derive Marcinkiewicz-Zygmund (MZ) inequalities for $\Pi_{n}^{q}$, which in turn will help us to establish the existence of the quadrature formulas. To do this, we first need to discuss doubling weights, which were introduced by Mastroianni and Totik [12, and their connection with MarkovBernstein inequalities for trigonometric polynomials. These Markov-Bernstein inequalities will lead to the MZ inequalities for $\mathbb{S}^{q}$, described in Theorem 3.1

3.1. Doubling weights. Following Mastroianni and Totik [12], a $2 \pi$-periodic, integrable, and nonnegative function $W$ will be called a doubling weight if for any $x \in[-\pi, \pi]$ and $\rho>0$,

$$
\int_{x-2 / \rho}^{x+2 / \rho} W(t) d t \leq 2^{\gamma} \int_{x-1 / \rho}^{x+1 / \rho} W(t) d t .
$$

For example, for any $\kappa>-1,|\sin t|^{\kappa}$ is a doubling weight. Moving averages of doubling weights will play an important role here. Consider any interval $I=$ $[x-1 / \rho, x+1 / \rho]$. We associate with $W$ the following family of moving averages,

$$
W_{\rho}(x):=\frac{1}{|I|} \int_{I} W d t=\frac{\rho}{2} \int_{x-1 / \rho}^{x+1 / \rho} W(t) d t .
$$

By multiplying both sides of (3.1) by $\rho / 4$, we obtain an equivalent definition in terms of moving averages,

$$
W_{\rho / 2}(x) \leq 2^{\gamma-1} W_{\rho}(x) .
$$

We need the following results due to Erdélyi [4].

Proposition 3.1. Let $W$ be a doubling weight, $\rho>0, W_{\rho}$ be as in 3.2), $0<p<$ $\infty$, and $T$ be a trigonometric polynomial. The constants used here, denoted simply by $C$, depend only on $\gamma$.

(a) For every $x, y \in \mathbb{R}$,

$$
\frac{W_{\rho}(x)}{(2+2 \rho|x-y|)^{\gamma}} \leq W_{\rho}(y) \leq(2+2 \rho|x-y|)^{\gamma} W_{\rho}(x) .
$$


(b) If $\operatorname{deg} T \leq \rho$, then

$$
\frac{\int_{-\pi}^{\pi}|T(t)|^{p} W(t) d t}{C(1+p)^{C}} \leq \int_{-\pi}^{\pi}|T(t)|^{p} W_{\rho}(t) d t \leq C(1+p)^{C} \int_{-\pi}^{\pi}|T(t)|^{p} W(t) d t .
$$

(c) The following Bernstein inequalities hold for any $T$ :

$$
\int_{-\pi}^{\pi}\left|T^{\prime}(t)\right|^{p} W(t) d t \leq C^{1+p}(\operatorname{deg} T)^{p} \int_{-\pi}^{\pi}|T(t)|^{p} W(t) d t .
$$

Remark. In 4], an integer $n$ was used instead of the continuous variable $\rho$. However, it is straightforward to show that if $n \leq \rho \leq n+1$, then

$$
\frac{\rho}{n+1} W_{n+1}(x) \leq W_{\rho}(x) \leq \frac{n}{\rho} W_{n}(x) .
$$

Hence, we may use the continuous variable $\rho$ in place of the integer $n$.

We conclude our discussion of doubling weights with the following lemma.

Lemma 3.1. Let $W$ be a doubling weight, $I$ be a closed interval, and $g: I \rightarrow[0, \infty)$ be integrable and have $I$ as its support. If $|I| \leq 2 \rho^{-1}$, then

$$
\int_{I} g(t) d t \int_{I} W(u) d u \leq 2^{2 \gamma+1} \rho^{-1} \int_{I} g(t) W_{\rho}(t) d t
$$

Proof. Regard $g$ as a weight function. Since $W_{\rho}$ is continuous, the mean value theorem for integrals implies that there exists $\bar{t} \in I$ such that

$$
W_{\rho}(\bar{t})=\frac{\int_{I} g(t) W_{\rho}(t) d t}{\int_{I} g(t) d t}
$$

If $t_{c}$ is the center of the interval $I$, then

$$
\int_{I} W(t) d t \leq \int_{t_{c}-1 / \rho}^{t_{c}+1 / \rho} W(t) d t=\frac{2}{\rho} W_{\rho}\left(t_{c}\right)
$$

and $\left|t_{c}-\bar{t}\right|<|I| / 2<\rho^{-1}$. By (3.4), we have

$$
\int_{I} W(t) d t \leq \frac{2}{\rho} W_{\rho}\left(t_{c}\right) \leq \frac{2}{\rho}(2+2)^{\gamma} W_{\rho}(\bar{t})=\frac{2^{2 \gamma+1} \rho^{-1} \int_{I} g(t) W_{\rho}(t) d t}{\int_{I} g(t) d t} .
$$

The result follows on multiplying both sides by $\int_{I} g(t) d t$ and combining the inequalities above.

3.2. Scattered points and compatible decompositions. We now want to consider a finite subset $\mathcal{C}_{0} \subset \mathbb{S}^{q}$ comprising distinct, scattered points. This set will provide the sites required in the quadrature formulas we will derive later. The mesh norm for $\mathcal{C}_{0}$ is defined by

$$
\delta_{\mathcal{C}_{0}}:=\max _{\mathbf{x} \in \mathbb{S}^{q}} \operatorname{dist}\left(\mathbf{x}, \mathcal{C}_{0}\right):=\max _{\mathbf{x} \in \mathbb{S}^{q}} \min _{\mathbf{y} \in \mathcal{C}_{0}} \operatorname{dist}(\mathbf{x}, \mathbf{y}),
$$

where $\operatorname{dist}(\mathbf{x}, \mathbf{y}):=\arccos (\mathbf{x} \cdot \mathbf{y})$ is the geodesic distance between $\mathbf{x}, \mathbf{y} \in \mathbb{S}^{q}$.

In addition to these sites, we need a decomposition $\mathcal{R}$ for $\mathbb{S}^{q}$.

Definition 3.1. Let $\mathcal{R}$ be a finite collection of closed, nonoverlapping (i.e., having no common interior points) regions $R \subset \mathbb{S}^{q}$ such that $\bigcup_{R \in \mathcal{R}} R=\mathbb{S}^{q}$. We will say that $\mathcal{R}$ is $\mathcal{C}_{0}$-compatible if each $R \in \mathcal{R}$ contains at least one point of $\mathcal{C}_{0}$ in its interior. The partition norm for $\mathcal{R}$ is defined by

$$
\|\mathcal{R}\|:=\max _{R \in \mathcal{R}} \operatorname{diam} R \text {. }
$$


If $\mathcal{R}$ is a $\mathcal{C}_{0}$-compatible decomposition, we can choose one point $\xi \in \mathcal{C}_{0}$ interior to each region. We can then use this point to label uniquely the region as $R_{\xi}$; the set of such points will be denoted by $\mathcal{C}$. Of course, $\mathcal{C} \subseteq \mathcal{C}_{0}$ and $\mathcal{R}=\left\{R_{\xi}\right\}_{\xi \in \mathcal{C}}$. Furthermore, no point in $R_{\xi}$ can be farther from $\xi$ than diam $R_{\xi} \leq\|\mathcal{R}\|$; hence, $\delta_{\mathcal{C}_{0}}<\|\mathcal{R}\|$. Moreover, it is also easy to see that $\mathcal{R}$ is $\mathcal{C}$-compatible, so $\delta_{\mathcal{C}}<\|\mathcal{R}\|$. Finally, removing points from $\mathcal{C}_{0}$ only increases its mesh norm; hence, we have the bounds

$$
\delta_{\mathcal{C}_{0}} \leq \delta_{\mathcal{C}}<\|\mathcal{R}\|
$$

Once we have found a $\mathcal{C}_{0}$-compatible decomposition $\mathcal{R}$, we will make use only of the points in the reduced $\operatorname{set} \mathcal{C}$. For that reason, we will work only with $\mathcal{C}$. Using the remaining points from $\mathcal{C}_{0}$ will have no effect on how well our quadrature formulas behave for smooth functions. They can, however, be used to average values in a region and reduce noise. We will not address this topic here.

A $\mathcal{C}_{0}$-compatible decomposition $\mathcal{R}$ serves to cluster dense subsets in $\mathcal{C}_{0}$ into regions, so that such subsets can be treated as units. The interior point of $R$ then represents the cluster. For $q \geq 2$, it is possible to use a boundary point in $\mathcal{C}_{0} \cap R$ for the same purpose. However, ambiguities arise, and little is gained by it. In the $q=1$-case, the circle, no ambiguities arise, since the regions are closed intervals in $[-\pi, \pi]$, and boundary points are just endpoints of such intervals. We make use of this fact in the proof of Corollary 4.1

In our next result, we will show that for every $\mathcal{C}_{0}$ we can always construct a $\mathcal{C}_{0}$-compatible decomposition $\mathcal{R}$ for which $\|\mathcal{R}\|, \delta_{\mathcal{C}_{0}}, \delta_{\mathcal{C}},|\mathcal{C}|^{-\frac{1}{q}}$ are all comparable. This will be very important in what follows.

To do this, we will make use of regions that are spherical simplices. For $q=2$, these are just spherical triangles, and for $q=3$ they are spherical tetrahedra. See Flanders' book [5, §5.6] for more details.

Proposition 3.2. If $\mathcal{C}_{0}$ and $\delta_{\mathcal{C}_{0}}$ are as above, then there exists a $\mathcal{C}_{0}$-compatible decomposition $\mathcal{R}$ for which each $R \in \mathcal{R}$ is a spherical simplex. A reduced set $\mathcal{C}$ can be found with each point $\xi \in \mathcal{C}$ in the interior of $R_{\xi}$. In addition, the norm of $\mathcal{R}$, the mesh norm $\delta_{\mathcal{C}_{0}}$, and the common cardinality of $\mathcal{R}$ and $\mathcal{C}$ satisfy

$$
\begin{aligned}
2 \delta_{\mathcal{C}_{0}} \leq\|\mathcal{R}\| & \leq 8 q \sqrt{2 q(q+1)} \delta_{\mathcal{C}_{0}} \\
|\mathcal{R}|=|\mathcal{C}| & =2^{q+1}\left\lfloor\frac{1}{2 q \sqrt{q+1} \delta_{\mathcal{C}_{0}}}\right\rfloor^{q} \\
\frac{2}{\sqrt{q(q+1)}}(|\mathcal{C}| / 2)^{-\frac{1}{q}} \leq\|\mathcal{R}\| & \leq 4 \sqrt{2 q}(|\mathcal{C}| / 2)^{-\frac{1}{q}}
\end{aligned}
$$

Further, for any $R \in \mathcal{R}$ and $\mathbf{x}$ in the interior of $R$, any geodesic through $\mathbf{x}$ intersects $R$ in exactly two points.

Proof. First, we illustrate the proof for the 2-sphere, where slightly better constants can be obtained. Divide the sphere into octants; these are of course bounded by geodesics (arcs of great circles). Consider the first octant, $\mathcal{O}_{I}$. Let $\mathbf{u}=\left(\begin{array}{lll}1 & 1 & 1\end{array}\right)$. If $\mathbf{x} \in \mathcal{O}_{I}$, then $X=\frac{\mathbf{x}}{\mathbf{x} \cdot \mathbf{u}}$ sets up a one-to-one correspondence between points in $\mathcal{O}_{I}$ and the (equilateral) triangle $T_{I}$ formed by the points $\left(\begin{array}{lll}1 & 0 & 0\end{array}\right),\left(\begin{array}{lll}0 & 1 & 0\end{array}\right)$, and $\left(\begin{array}{lll}0 & 0 & 1\end{array}\right)$. The most important feature of this correspondence is that the geodesic joining two points $\mathbf{x} \in \mathcal{O}_{I}$ and $\mathbf{y} \in \mathcal{O}_{I}$ corresponds to the line segment joining $X$ and $Y$ on $T_{I}$, because all four points lie on a plane through the origin. Moreover, with a little 
work, one sees that if $\theta \in[0, \pi / 2]$ is the geodesic distance between $\mathbf{x}, \mathbf{y} \in \mathcal{O}_{I}$, then

$$
\frac{1}{2} \theta \leq \frac{2}{\sqrt{3}} \sin (\theta / 2) \leq\|X-Y\| \leq 4 \sin (\theta / 2) \leq 2 \theta,
$$

where $\|X-Y\|$ denotes the Euclidean distance between $X$ and $Y$. Divide $T_{I}$ into $N^{2}$ congruent equilateral triangles, with sides of length $L=\sqrt{2} / N$. Each of these triangles contains an inscribed circle of radius $L /(2 \sqrt{3})$. The corresponding spherical triangles thus contain a spherical cap of radius $L /(4 \sqrt{3})$. They are no longer equilateral, but their sides have lengths less than $2 L$. Now, choose $N=$ $\left\lfloor\left(2 \sqrt{6} \delta_{\mathcal{C}_{0}}\right)^{-1}\right\rfloor$, so that $N \leq\left(2 \sqrt{6} \delta_{C}\right)^{-1}<N+1$, so that $\delta_{\mathcal{C}_{0}} \leq(2 \sqrt{6} N)^{-1}=$ $L /(4 \sqrt{3})$.

Repeat this procedure for each octant. If necessary, rotate the set of triangles to ensure that no point of $\mathcal{C}_{0}$ lies on the boundary of one of the spherical triangles. Since no point of the sphere is at a distance greater than $\delta_{\mathcal{C}_{0}}$ from $\mathcal{C}_{0}$, and since each spherical triangle contains a cap of radius $L /(4 \sqrt{3}) \geq \delta_{\mathcal{C}_{0}}$, with no points of the cap lying on the edge of the triangle, there must be a point, $\xi$, interior to the triangle that is in $\mathcal{C}_{0}$. Label the triangle (interior plus boundary) as $R_{\xi}$. Note that $2 \delta_{\mathcal{C}_{0}}<\operatorname{diam} R_{\xi} \leq 2 L \leq 8 \sqrt{3} \delta_{\mathcal{C}_{0}}$. Since a geodesic passing through the interior of an $R_{\xi}$ corresponds to a straight line intersecting the corresponding planar triangle, it will intersect $\partial R_{\xi}$ twice. Thus, $\left\{R_{\xi}\right\}$ is $\mathcal{C}_{0}$-compatible, and (3.10) holds with $8 \sqrt{3} \delta_{\mathcal{C}_{0}}$ on the right hand side instead of the stated estimate. Concerning $|\mathcal{R}|$, note that the number of spherical triangles per octant is $N^{2}=\left\lfloor\left(2 \sqrt{6} \delta_{\mathcal{C}_{0}}\right)^{-1}\right\rfloor^{2}$, so there are $8\left\lfloor\left(2 \sqrt{6} \delta_{\mathcal{C}_{0}}\right)^{-1}\right\rfloor^{2}$ in the decomposition and in the reduced set $\mathcal{C}$. For the last set of bounds involving $|\mathcal{C}|$, see the general case below.

The $q$-dimensional sphere, $\mathbb{S}^{q}$, may be dealt with similarly. $\mathbb{S}^{q}$ is divided into $2^{q+1}$ spherical simplices analogous to octants. Let $\mathcal{O}_{I}$ be the spherical simplex on which all the coordinates of $x \in \mathbb{S}^{q} \subset \mathbb{R}^{q+1}$ are nonnegative, and let $T_{I}=$ $\left\{\sum_{j=1}^{q+1} t_{j} e_{j} \mid t_{j} \in[0,1], \sum_{j} t_{j}=1\right\}$ be the (equilateral) Euclidean simplex formed from the standard set of unit vectors in $\mathbb{R}^{q+1},\left\{e_{j}\right\}_{j=1}^{q+1}$. As before, one can easily show how distances on the spherical simplices are related to distances on the corresponding Euclidean simplices; namely, if $\theta \in[0, \pi / 2]$ is the geodesic distance between $\mathbf{x}, \mathbf{y} \in \mathcal{O}_{I}$, then

$$
\frac{1}{2 \sqrt{q}} \theta \leq \frac{2 \sin (\theta / 2)}{\sqrt{q+1}} \leq\|X-Y\| \leq 2 \sqrt{q+2} \sin (\theta / 2) \leq 2 \sqrt{q} \theta .
$$

It is straightforward to show that an equilateral Euclidean simplex, with $L$ being the length of a side, contains a sphere of radius $L / \sqrt{2 q(q+1)}$. The corresponding spherical simplex then contains a cap of radius $L /(2 q \sqrt{2(q+1)})$. Let $L=\sqrt{2} / N$. Taking $N=\left\lfloor\left(1 /(2 q \sqrt{q+1}) \delta_{\mathcal{C}_{0}}\right)\right\rfloor$ gives us a radius larger than $\delta_{\mathcal{C}_{0}}$, so $2 \delta_{\mathcal{C}_{0}} \leq$ $\operatorname{diam} R_{\xi}$. Moreover,

$$
\operatorname{diam} R_{\xi} \leq 2 \sqrt{q} L=\frac{2 \sqrt{2 q}}{N} \leq \frac{4 \sqrt{2 q}}{N+1} \leq 8 q \sqrt{2 q(q+1)} \delta_{\mathcal{C}_{0}} .
$$

Hence, $\mathcal{R}$ is a $\mathcal{C}_{0}$-compatible decomposition, which satisfies also the condition regarding the choice of a reduced subset and the geodesic condition. Further, (3.10) holds. The number of simplices in one "octant" is $N^{q}$, and so the cardinality of $\mathcal{R}$ is $2^{q+1}\left\lfloor\left(1 /(2 q \sqrt{q+1}) \delta_{\mathcal{C}_{0}}\right)\right\rfloor^{q}$. Since there is one point in $\mathcal{C}$ per simplex, this is also equal to $|\mathcal{C}|$. This proves (3.11). 
To obtain the bounds involving $|\mathcal{C}|$, observe that $2 N=(|\mathcal{C}| / 2)^{1 / q}$, and so the right side of the third inequality follows from $\|\mathcal{R}\| \leq \frac{2 \sqrt{2 q}}{N}$. For the left side, note that each spherical simplex contains a spherical cap of radius $L /(2 q \sqrt{2(q+1)})=$ $1 /(2 q \sqrt{(q+1)} N)$, so $2 /(q \sqrt{(q+1)} 2 N) \leq\|\mathcal{R}\|$. Replacing $2 N$ by $(|\mathcal{C}| / 2)^{1 / q}$ completes the proof.

We point out that in the $\mathbb{S}^{q}$ case, we used somewhat cruder bounds than in the $q=2$ demonstration. This results in somewhat different bounds when we reduce the numbers in the general $q$ case to $q=2$.

3.3. Fundamental estimate. Let $V(\cdot)$ be a univariate polynomial having degree at least $n$. In addition, we require that for every $P \in \Pi_{n}^{q}$, we have

$$
P(\mathbf{x})=\int_{\mathbb{S} q} V(\mathbf{x} \cdot \mathbf{y}) P(\mathbf{y}) d \mu_{q}(\mathbf{y}) .
$$

In other words, $V$ is a reproducing kernel for $\Pi_{n}^{q}$. We wish to estimate the quantity

$$
E_{\mathcal{C}}(P):=\left|\int_{\mathbb{S} q}\right| P(\mathbf{x})\left|d \mu_{q}(\mathbf{x})-\sum_{\xi \in \mathcal{C}}\right| P(\xi)\left|\mu_{q}\left(R_{\xi}\right)\right| .
$$

From the triangle inequality and (3.13), one has that

$$
\begin{aligned}
E_{\mathcal{C}}(P) & \leq \sum_{\xi \in \mathcal{C}} \int_{R_{\xi}}|P(\mathbf{x})-P(\xi)| d \mu_{q}(\mathbf{x}) \\
& \leq\|P\|_{\mathbb{S} q, 1} \sup _{\mathbf{y} \in \mathbb{S}^{q}}\left(\sum_{\xi \in \mathcal{C}} \int_{R_{\xi}}|V(\mathbf{x} \cdot \mathbf{y})-V(\xi \cdot \mathbf{y})| d \mu_{q}(\mathbf{x})\right) .
\end{aligned}
$$

We want to estimate the sum on the right above.

Consider the standard parametrization of $\mathbb{S}^{q}$ embedded in $\mathbb{R}^{q+1}$ in terms of the angles $\theta_{1}, \ldots, \theta_{q}$, where $-\pi \leq \theta_{1} \leq \pi$ and $0 \leq \theta_{k} \leq \pi$ for $k=2, \ldots, q$. If $\mathbf{x} \in \mathbb{S}^{q}$, then the $k$ th component of $\mathbf{x}$ is given by

$$
x_{k}= \begin{cases}\prod_{j=1}^{q} \sin \theta_{j} & k=1, \\ \cos \theta_{k-1} \prod_{j=k}^{q} \sin \theta_{j} & 2 \leq k \leq q, \\ \cos \theta_{q} & k=q+1 .\end{cases}
$$

The measure $\mu_{q}$ on $\mathbb{S}^{q}$ can be expressed in these coordinates as

$$
d \mu_{q}(\mathbf{x})=\prod_{k=1}^{q} \sin ^{k-1}\left(\theta_{k}\right) d \theta_{k}
$$

Note that

$$
d \mu_{q}=\sin ^{q-1}\left(\theta_{q}\right) d \theta_{q} d \mu_{q-1},
$$

and that one can extend $\sin ^{q-1}\left(\theta_{q}\right)$ to the even, $2 \pi$-periodic doubling weight $W(\cdot)=|\sin (\cdot)|^{q-1}$.

Proposition 3.3. Let $\mathcal{R}$ be a $\mathcal{C}$-compatible decomposition of $\mathbb{S}^{q}$, with mesh norm $\|\mathcal{R}\|$. If $V(\cdot)$ is a univariate polynomial for which $\operatorname{deg} V \leq(2\|\mathcal{R}\|)^{-1}$, then

$$
\sup _{\mathbf{y} \in \mathbb{S}^{q}}\left\{\sum_{\xi \in \mathcal{C}} \int_{R_{\xi}}|V(\mathbf{x} \cdot \mathbf{y})-V(\xi \cdot \mathbf{y})| d \mu_{q}(\mathbf{x})\right\} \leq C_{q}\|\mathcal{R}\| \operatorname{deg} V\|V\|_{w_{q}, 1},
$$


where $C_{q}$ depends only on the dimension $q$.

Proof. Fix a point $\mathbf{y} \in \mathbb{S}^{q}$ and use spherical coordinates, with the point $\mathbf{y}$ taken to be the north pole. If we set $\theta=\theta_{q}$ in (3.16) , then $\mathbf{x} \cdot \mathbf{y}=\cos \theta$ and $\mathbf{y} \cdot \xi=\cos \theta_{\xi}$, and

$$
I_{\xi}:=\int_{R_{\xi}}|V(\mathbf{x} \cdot \mathbf{y})-V(\xi \cdot \mathbf{y})| d \mu_{q}(\mathbf{x})=\int_{R_{\xi}}\left|\int_{\theta_{\xi}}^{\theta} \frac{d}{d t} V(\cos t) d t\right| d \mu_{q}(\mathbf{x}) .
$$

If $\theta^{ \pm}$are the low and high values for the coordinate $\theta_{q}$ in the region $R_{\xi}$, then we have

$$
I_{\xi} \leq \mu_{q}\left(R_{\xi}\right) \int_{\theta^{-}}^{\theta^{+}}\left|\frac{d}{d t} V(\cos t)\right| d t .
$$

Of course, $\theta_{\xi} \in\left[\theta^{-}, \theta^{+}\right]$, because $\xi$ is in $R_{\xi}$.

The next step is to cover the sphere with overlapping "bands". Take $N=\left\lfloor\frac{\pi}{\|\mathcal{R}\|}\right\rfloor$. For $k=1, \ldots, N$, let $J_{k}:=[(k-1) \pi / N, k \pi / N]$, and for $k=1, \ldots, N-1$, define $B_{k}$ to be all $\mathbf{x} \in \mathbb{S}^{q}$ with $\theta_{q} \in J_{k} \cup J_{k+1}$. The common length for each interval is $\pi / N \geq\|\mathcal{R}\| \geq \operatorname{diam}\left(R_{\xi}\right) \geq \theta^{+}-\theta^{-}$. Thus, if $\theta^{-} \in J_{k}$, then $\left[\theta^{-}, \theta^{+}\right] \subset J_{k} \cup J_{k+1}$ or, when $k=N-1,\left[\theta^{-}, \theta^{+}\right] \subset J_{N-1} \subset J_{N-2} \cup J_{N-1}$. It follows that when $\theta^{-} \in J_{k}$, we have $R_{\xi} \subset B_{k}$ and that the bound in equation (3.20) may be replaced by

$$
I_{\xi} \leq \mu_{q}\left(R_{\xi}\right) \int_{(k-1) \pi / N}^{(k+1) \pi / N}\left|\frac{d}{d t} V(\cos t)\right| d t .
$$

Note that the $R_{\xi}$ 's are nonoverlapping (no common interior points), so that we have $\sum_{R_{\xi} \subset B_{k}} \mu_{q}\left(R_{\xi}\right) \leq \mu_{q}\left(B_{k}\right)$. Summing $I_{\xi}$ from all $R_{\xi} \subset B_{k}$ then yields

$$
\sum_{R_{\xi} \subset B_{k}} I_{\xi} \leq \mu_{q}\left(B_{k}\right) \int_{(k-1) \pi / N}^{(k+1) \pi / N}\left|\frac{d}{d t} V(\cos t)\right| d t .
$$

Since each $R_{\xi}$ is contained in at least one band, summing over the contribution from each band majorizes $\sum_{\xi \in \mathcal{C}} I_{\xi}$. Combining this with the previous estimate yields the bound,

$$
\sum_{\xi \in \mathcal{C}} I_{\xi} \leq \sum_{k=1}^{N-1} \mu_{q}\left(B_{k}\right) \int_{(k-1) \pi / N}^{(k+1) \pi / N}\left|\frac{d}{d t} V(\cos t)\right| d t
$$

Moreover, the volume of $B_{k}$ is given by $\mu_{q}\left(B_{k}\right)=\omega_{q-1} \int_{(k-1) \pi / N}^{(k+1) \pi / N} W(t) d t$, where $W(t)=|\sin (t)|^{q-1}$ extends $\sin ^{q-1}(t)$ to be an even doubling weight. After inserting this in the bound above, we obtain

$$
\sum_{\xi \in \mathcal{C}} I_{\xi} \leq \sum_{k=1}^{N-1} \omega_{q-1} \int_{(k-1) \pi / N}^{(k+1) \pi / N} W(t) d t \int_{(k-1) \pi / N}^{(k+1) \pi / N}\left|\frac{d}{d t} V(\cos t)\right| d t .
$$

We now apply Lemma 3.1 to the product of the two integrals in equation 3.21. Choose $\rho=(2\|\mathcal{R}\|)^{-1}<N / \pi$. From (3.21) and (3.7), we arrive at

$$
\begin{aligned}
\sum_{\xi \in \mathcal{C}} I_{\xi} & \leq 2^{2 \gamma+1} \rho^{-1} \omega_{q-1} \sum_{k=1}^{N-1} \int_{(k-1) \pi / N}^{(k+1) \pi / N}\left|\frac{d}{d t} V(\cos t)\right| W_{\rho}(t) d t \\
& \leq 2^{2 \gamma+1} \rho^{-1} \omega_{q-1} \int_{0}^{\pi}\left|\frac{d}{d t} V(\cos t)\right| W_{\rho}(t) d t
\end{aligned}
$$


It is easy to check that $W$, being even in $t$, implies $W_{\rho}$ is even as well. Moreover, the function $\left|\frac{d}{d t} V(\cos t)\right|$ is also even, and $\frac{d}{d t} V(\cos t)$ is a trigonometric polynomial. Thus, by (3.5) and (3.6) with $p=1$ and our assumption that $\rho=(2\|\mathcal{R}\|)^{-1} \geq \operatorname{deg} V$ we have

$$
\begin{aligned}
\int_{0}^{\pi}\left|\frac{d}{d t} V(\cos t)\right| W_{\rho}(t) d t & =\frac{1}{2} \int_{-\pi}^{\pi}\left|\frac{d}{d t} V(\cos t)\right| W_{\rho}(t) d t \\
& \leq \frac{1}{2} \tilde{C} \int_{-\pi}^{\pi}\left|\frac{d}{d t} V(\cos t)\right| W(t) d t \\
& \leq \frac{\operatorname{deg} V}{2} C \int_{-\pi}^{\pi}|V(\cos t)| W(t) d t \\
& \leq C \operatorname{deg} V \int_{0}^{\pi}|V(\cos t)| W(t) d t .
\end{aligned}
$$

Putting this together with the previous inequality, with $W(t)=\sin ^{q-1}(t)$ on $[0, \pi]$, and using $\rho=(2\|\mathcal{R}\|)^{-1}$, we see that

$$
\begin{aligned}
\sum_{\xi \in \mathcal{C}} I_{\xi} & \leq 2^{2 \gamma+2} \omega_{q-1}\|\mathcal{R}\|(\operatorname{deg} V) C \int_{0}^{\pi}|V(\cos t)| \sin ^{q-1}(t) d t \\
& =2^{2 \gamma+2} \omega_{q-1}\|\mathcal{R}\|(\operatorname{deg} V) C\|V\|_{w_{q}, 1} .
\end{aligned}
$$

Both $\gamma$ and $C$ above depend on the doubling weight, which in turn depends on the dimension $q$. Finally, letting $C_{q}=2^{2 \gamma+2} C \omega_{q-1}$ we obtain the desired inequality.

Recall that in $\$ 2.1$, we discussed the delayed-mean kernels $\tau_{n}^{(k)}$. When $k>\frac{q-1}{2}$ is an integer, and $n \geq N_{q}$, these $((k+1) n-1)$-degree polynomials are reproducing kernels on $\Pi_{n}^{q}$ (cf. Corollary 2.2). If $k=k_{q}:=\lfloor(q-1) / 2\rfloor+1$, the condition on $k$ is satisfied. Moreover, by Corollary 2.2, the norms $\left\|\tau_{n}^{\left(k_{q}\right)}\right\|_{w_{q}, 1}$ are uniformly bounded in $n$. Thus, for $n \geq N_{q}$, we may use $V=\tau_{n}^{\left(k_{q}\right)}$ and $\operatorname{deg} V=\left(k_{q}+1\right) n-1$ in Proposition 3.3 and apply the inequality there in (3.15) to obtain the following estimate on $E_{\mathcal{C}}(P)$.

Corollary 3.1. Take $V=\tau_{n}^{\left(k_{q}\right)}$ to be the reproducing kernel for $\Pi_{n}^{q}, n \geq N_{q}$. If $\mathcal{R}$ is any $\mathcal{C}$-compatible decomposition for which $\|\mathcal{R}\|<\frac{1}{2\left(k_{q}+1\right) n}$, then for all $P \in \Pi_{n}^{q}$ we have

$$
E_{\mathcal{C}}(P) \leq \sum_{\xi \in \mathcal{C}} \int_{R_{\xi}}|P(x)-P(\xi)| d \mu_{q}(x) \leq \widetilde{C}_{q}\left(k_{q}+1\right) n\|\mathcal{R}\|\|P\|_{\mathbb{S}^{q}, 1},
$$

where $E_{C}(P)$ is defined in (3.14) and $\widetilde{C}_{q}:=C_{q} \sup _{n \geq N_{q}}\left\{\left\|\tau_{n}^{\left(k_{q}\right)}\right\|_{w_{q}, 1}\right\}$.

We remark that the case of the circle $(q=1)$ is simpler, and can be handled without using the reproducing kernel $V$ or the theory of doubling weights. The key estimate,

$$
\int_{R_{\xi}}|P(\theta)-P(\xi)| d \theta \leq \operatorname{diam}\left(R_{\xi}\right) \int_{R_{\xi}}\left|P^{\prime}(\theta)\right| d \theta,
$$

is easy to establish. If we sum over the $\xi$ 's, use the $L^{1}$ Bernstein inequality [21, $\left\|P^{\prime}\right\|_{\mathbb{S}^{1}, 1} \leq n\|P\|_{\mathbb{S}^{1}, 1}$, and take the maximum diameter above, then we arrive at the following sharper result. 
Corollary 3.2. In the case of the circle $(q=1)$,

$$
E_{\mathcal{C}}(P) \leq \sum_{\xi \in \mathcal{C}} \int_{R_{\xi}}|P(\theta)-P(\xi)| d \theta \leq n\|\mathcal{R}\|\|P\|_{\mathbb{S}^{1}, 1}, \quad P \in \Pi_{n}^{1} .
$$

3.4. $L^{p}$ Marcinkiewicz-Zygmund inequalities. The estimate (3.23) will lead to the Marcinkiewicz-Zygmund inequalities for $\mathbb{S}^{q}$ (see Theorem 3.1 below). Using the notation from the Corollary 3.1, define the quantity

$$
\varrho_{q}:=\min \left\{\frac{1}{2\left(k_{q}+1\right)}, \frac{1}{\left(k_{q}+1\right) \widetilde{C}_{q}}\right\} .
$$

For future reference, note that from (2.16), $k_{q} \geq 1$, and, consequently,

$$
\varrho_{q} \leq 1
$$

Theorem 3.1. Let $\mathcal{R}$ be a $\mathcal{C}$-compatible decomposition, and let $N_{q}$ be as in Theorem 2.1. If $n \geq N_{q}, \eta \in(0,1)$, and the norm $\|\mathcal{R}\|$ satisfies

$$
\|\mathcal{R}\|<\frac{\eta}{n} \varrho_{q},
$$

where $\varrho_{q}$ is given by (3.25), then for all $P \in \Pi_{n}^{q}$

$$
(1-\eta)\|P\|_{\mathbb{S} q, p} \leq\left\|\left.P\right|_{\mathcal{C}}\right\|_{\mathcal{C}, p} \leq(1+\eta)\|P\|_{\mathbb{S} q, p},
$$

where $\|\cdot\|_{\mathcal{C}, p}$ is the weighted $\ell^{p}$ norm defined for all $f: \mathcal{C} \rightarrow \mathbb{R}$ by

$$
\|f\|_{\mathcal{C}, p}:= \begin{cases}\left(\sum_{\xi \in \mathcal{C}}|f(\xi)|^{p} \mu_{q}\left(R_{\xi}\right)\right)^{1 / p} & \text { if } 1 \leq p<\infty, \\ \sup _{\xi \in \mathcal{C}}\{|f(\xi)|\} & \text { if } p=\infty .\end{cases}
$$

Proof. Using (3.14), (3.23), and (3.27), we deduce that if $P \in \Pi_{n}^{q}$, then

$$
\left|\|P\|_{\mathbb{S}^{q}, 1}-\sum_{\xi \in \mathcal{C}}\right| P(\xi)\left|\mu_{q}\left(R_{\xi}\right)\right|<\eta\|P\|_{\mathbb{S}^{q}, 1},
$$

which in turn implies (3.28) with $p=1$.

Using a Markov inequality established by Bos et al. 1], Jetter, Stöckler and Ward 10] implicitly obtained (3.28) in the case $p=\infty$ under the condition that $\delta_{\mathcal{C}}<\eta / n$. By (3.9) and (3.26), we see that if $\|\mathcal{R}\| \leq(\eta / n) \varrho_{q}$, then $\delta_{\mathcal{C}}<\eta / n$. Hence, (3.28) also holds under the stated condition on $\|\mathcal{R}\|$.

Next, we consider the "intermediate" cases. Let $T:\left.\Pi_{n}^{q} \rightarrow \Pi_{n}^{q}\right|_{\mathcal{C}} \subset \mathbb{R}^{|\mathcal{C}|}$ be the linear transformation defined via $T(P)=\left.P\right|_{\mathcal{C}}$, where $\|\cdot\|_{\mathbb{S}^{q}, p}$ is used as the norm for $\Pi_{n}^{q}$ and $\|\cdot\|_{\mathcal{C}, p}$ is that for $\mathbb{R}^{|\mathcal{C}|}$. Since the spaces involved are finite dimensional, the operator norm $\|T\|_{p}<\infty$ for $1 \leq p \leq \infty$. By [3, Theorem VI.10.10, p. 524], $\|T\|_{p}$ is a convex function of $1 / p$. Thus, $\|T\|_{p} \leq \max \left\{\|T\|_{1},\|T\|_{\infty}\right\}$, and so, from the already proved estimates (3.28) in the cases $p=1$ and $p=\infty$, we have $\|T\|_{p} \leq 1+\eta$. This establishes the right side of (3.28).

To get the left side, note that the spaces $\Pi_{n}^{q}$ and $T\left(\Pi_{n}^{q}\right)=\left.\Pi_{n}^{q}\right|_{\mathcal{C}}$ are isomorphic, so $T^{-1}:\left.\Pi_{n}^{q}\right|_{\mathcal{C}} \rightarrow \Pi_{n}^{q} \subset L^{p}\left(\mathbb{S}^{q}\right)$ exists. Reversing the roles of the two spaces in the argument above then yields the desired inequality.

In the case when $q=1$, if one uses (3.24) from Corollary 3.2 instead of (3.14), then the result we get is sharper. Before stating it, we note that the MarcinkiewiczZygmund inequalities for the circle were obtained by many authors. In contrast to Erdelyi's version in [4, our estimates below do not require a minimal separation 
condition on the points, but they do require more points for a trigonometric polynomial of given degree.

Proposition 3.4. Let $q=1$ and let $P$ be a trigonometric polynomial of degree at most n. If $\|\mathcal{R}\|<\frac{\eta}{n}$, then (3.28) holds.

\section{Quadrature formulas}

We will now derive $L^{p}$ quadrature formulas that are exact for spherical harmonics of degree $n$, have positive weights, and require a number of evaluations roughly proportional to the dimension of the underlying space of spherical harmonics, $\Pi_{n}^{q}$. These results improve similar ones obtained in [11] in two important respects. First, the weights we give are guaranteed to be positive; the ones in [11] were of uncertain sign. Second, our results hold for all $L^{p}, 1 \leq p \leq \infty$. In [1], only the $L^{\infty}$ case was treated. To begin our derivation, we need to generalize slightly the notion of a norming set, which was central to the arguments in [10].

4.1. Norm generating sets. Let $X$ be a finite dimensional vector space with norm $\|\cdot\|_{X}$, and let $\mathcal{Z} \subset X^{*}$ be a finite set.

Definition 4.1. We will say that $\mathcal{Z}$ is a norm generating set for $X$ if the mapping $T_{\mathcal{Z}}: X \rightarrow \mathbb{R}^{|\mathcal{Z}|}$ defined by $T_{\mathcal{Z}}(x)=(z(x))_{z \in \mathcal{Z}}$ is injective. We will call $T_{\mathcal{Z}}$ the sampling operator.

We remark that if $\mathcal{V}:=T_{\mathcal{Z}}(X)$ is the range of $T_{\mathcal{Z}}$, then the injectivity of $T_{\mathcal{Z}}$ implies that $T_{\mathcal{Z}}^{-1}: \mathcal{V} \rightarrow X$ exists. Let $\mathbb{R}^{|\mathcal{Z}|}$ have a norm $\|\cdot\|_{\mathbb{R}^{|\mathcal{Z}|}}$, with $\|\cdot\|_{\left.\mathbb{R}^{\mid \mathcal{Z}}\right|^{*}}$ being its dual norm on $\mathbb{R}^{|\mathcal{Z}|^{*}}$. Equip $\mathcal{V}$ with the induced norm, and let $\left\|T_{\mathcal{Z}}^{-1}\right\|:=$ $\left\|T_{\mathcal{Z}}^{-1}\right\|_{\mathcal{V} \rightarrow X}$. In addition, let $\mathcal{K}_{+}$be the positive cone of $\mathbb{R}^{|\mathcal{Z}|}$; that is, all $\left(r_{z}\right) \in \mathbb{R}^{|\mathcal{Z}|}$ for which $r_{z} \geq 0$.

Proposition 4.1. Let $\mathcal{Z}$ be a norm generating set for $X$, with $T_{\mathcal{Z}}$ being the corresponding sampling operator. If $y \in X^{*}$, with $\|y\|_{X^{*}} \leq A$, then there exist real numbers $\left\{a_{z}\right\}_{z \in \mathcal{Z}}$, depending only on $y$ such that for every $x \in X$,

$$
y(x)=\sum_{z \in \mathcal{Z}} a_{z} z(x)
$$

and

$$
\left\|\left(a_{z}\right)\right\|_{\left.\mathbb{R}^{\mid \mathcal{Z}}\right|^{*}} \leq A\left\|T_{\mathcal{Z}}^{-1}\right\| .
$$

Also, if $\mathcal{V}$ contains an interior point $v_{0} \in \mathcal{K}_{+}$and if $y\left(T_{\mathcal{Z}}^{-1} v\right) \geq 0$ when $v \in \mathcal{V} \cap \mathcal{K}_{+}$, then we may choose $a_{\xi} \geq 0$ in (4.1).

Proof. We can define a linear functional $\tilde{y}$ on $\mathcal{V}$ via $\tilde{y}(v):=y\left(T_{\mathcal{Z}}^{-1} v\right)$. From this and $\|y\|_{X^{*}} \leq A$, we see that $\|\tilde{y}\|_{\mathcal{V}^{*}} \leq A\left\|T_{\mathcal{Z}}^{-1}\right\|$. By the Hahn-Banach theorem, $\tilde{y}$ has a norm-preserving extension to a functional $\tilde{y}_{\text {ext }}$ on $\mathbb{R}^{|\mathcal{Z}|}$. Hence, there exists $\left(a_{z}\right) \in \mathbb{R}^{|\mathcal{Z}|^{*}}$ such that for all $\left(r_{z}\right) \in \mathbb{R}^{|\mathcal{Z}|}$ we have

$$
\tilde{y}_{\text {ext }}\left(\left(r_{z}\right)\right)=\sum_{z \in \mathcal{Z}} a_{z} r_{z}, \quad\left\|\left(a_{z}\right)\right\|_{\mathbb{R}^{|\mathcal{Z}|^{*}}} \leq A\left\|T_{\mathcal{Z}}^{-1}\right\| .
$$

By construction, $y(x)=\tilde{y}(v)=\tilde{y}_{\text {ext }}(v)$, with $v=T_{\mathcal{Z}} x=(z(x))$. Consequently, using $v=T_{\mathcal{Z}} x$ in equation (4.3) gives equation (4.1), with (4.2) being the norm condition on $\left(a_{z}\right)$ from (4.3). The second part of the theorem is a consequence of the Krein-Rutman theorem [9, p. 20]. 
4.2. Scattered-site quadrature formulas for $\mathbb{S}^{q}$. By means of Theorem 3.1 and Proposition 4.1, we can deduce the following quadrature formula.

Theorem 4.1. Adopt the notation and assumptions of Theorem 3.1. Let $1 \leq p \leq$ $\infty$, and let $\|\cdot\|_{\mathcal{C}, p}$ be the weighted $\ell^{p}$ norm defined in $(3.29)$ for $\mathbb{R}^{|\mathcal{C}|}$. Then, if $\eta<\frac{1}{2}$, there exist nonnegative numbers $\left\{a_{\xi}\right\}_{\xi \in \mathcal{C}}$, such that for every $P \in \Pi_{n}^{q}$, we have

$$
\int_{\mathbb{S} q} P(\mathbf{x}) d \mu_{q}(\mathbf{x})=\sum_{\xi \in \mathcal{C}} a_{\xi} P(\xi)
$$

and

$$
\left\|\left(\frac{a_{\xi}}{\mu_{q}\left(R_{\xi}\right)}\right)\right\|_{\mathcal{C}, p^{\prime}} \leq \omega_{q}^{1 / p^{\prime}}(1-\eta)^{-1}, \quad 1 \leq p^{\prime}:=\frac{p}{p-1} \leq \infty .
$$

If we choose $\frac{1}{2} \leq \eta<1$, then (4.4) and (4.5) continue to hold, although the coefficients $\left\{a_{\xi}\right\}$ are no longer guaranteed to be nonnegative.

Proof. In Proposition 4.1. we take $X=\Pi_{n}^{q},\|P\|_{X}=\|P\|_{\mathbb{S}^{q}, p}$, and $\mathcal{Z}$ to be the set of point evaluation functionals $\left\{\delta_{\xi}\right\}_{\xi \in \mathcal{C}}$. The operator $T_{\mathcal{Z}}$ is then the restriction map $\left.P \mapsto P\right|_{\mathcal{C}}$, with $\|\cdot\|_{\mathbb{R}^{|\mathcal{C}|}}$ taken to be weighted $\ell^{p}$ norm $\|\cdot\|_{\mathcal{C}, p}$. The inequalities (3.28) give $\left\|T_{\mathcal{Z}}^{-1}\right\| \leq(1-\eta)^{-1}$. We now take $y$ to be the functional

$$
y: P \mapsto \int_{\mathbb{S}^{q}} P(\mathbf{x}) d \mu_{q}(\mathbf{x}) .
$$

By Hölder's inequality, $\|y\|_{X^{*}} \leq \omega_{q}^{1 / p^{\prime}}$. Proposition 4.1 now implies that (4.4) holds, subject to $\left\|\left(a_{\xi}\right)\right\|_{\mathbb{R}^{\mathcal{C}}} \leq \omega_{q}^{1 / p^{\prime}}(1-\eta)^{-1}$. Since $\left\|\left(a_{\xi}\right)\right\|_{\mathbb{R}^{\mathcal{C}} *}=\left\|\left(\frac{a_{\xi}}{\mu\left(R_{\xi}\right)}\right)\right\|_{\mathcal{C}, p^{\prime}}$, where $p^{\prime}:=p /(p-1)$, we also obtain 4.5.

To see that the $a_{\xi}$ 's may be chosen to be nonnegative, note that the constant polynomial $P_{0}(x)=1$ is in $\Pi_{n}^{q}$, and $v_{0}:=\left.P_{0}\right|_{\mathcal{C}}=(1,1, \ldots, 1)$ is an interior point of $\mathcal{K}_{+}$. Next, note that $T_{\mathcal{Z}} P=\left.P\right|_{\mathcal{C}}$ is in $\mathcal{V} \cap \mathcal{K}_{+}$if and only if $P(\xi) \geq 0$ for $\xi \in \mathcal{C}$. Observe that if $P(\xi) \geq 0$ for $\xi \in \mathcal{C}$, then using (3.23), (3.27), (3.28), we obtain

$$
\begin{aligned}
\left|y(P)-\left\|\left.P\right|_{\mathcal{C}}\right\|_{\mathcal{C}, 1}\right| & =\left|\sum_{\xi \in \mathcal{C}} \int_{\mathbb{S}^{q}}(P(x)-P(\xi)) d \mu_{q}(x)\right| \\
& \leq \sum_{\xi \in \mathcal{C}} \int_{\mathbb{S}^{q}}|P(x)-P(\xi)| d \mu_{q}(x) \\
& \leq \widetilde{C}_{q}\left(k_{q}+1\right) n\|\mathcal{R}\|\|P\|_{\mathbb{S}^{q}, 1} \\
& \leq \eta(1-\eta)^{-1}\left\|\left.P\right|_{\mathcal{C}}\right\|_{\mathcal{C}, 1} .
\end{aligned}
$$

Therefore,

$$
y(P) \geq \frac{1-2 \eta}{1-\eta}\left\|\left.P\right|_{\mathcal{C}}\right\|_{\mathcal{C}, 1}
$$

and $y(P)>0$ if $\eta<\frac{1}{2}$. Applying the last part of Proposition 4.1 then completes the proof.

Remark. The condition for the quadrature formula to hold is stated in terms of $\|\mathcal{R}\|$ in equation (3.27). Essentially, the condition states that $\|\mathcal{R}\| \sim n^{-1}$. In Proposition 3.2 we pointed out that it was possible to find $\mathcal{R}$ such that $\|\mathcal{R}\| \sim$ $|\mathcal{C}|^{-1 / q}$, where $|\mathcal{C}|$ is the cardinality of the (reduced) set $\mathcal{C}$. Thus, for this choice of 
$\mathcal{R}$, we have that $|\mathcal{C}| \sim n^{q}$, which is the order of the dimension of the space that we are exactly integrating.

It is worthwhile to examine the special case of the circle $(q=1)$. We suppose that we have a finite set $\mathcal{C}$ comprising $K$ distinct points that are located at angles $-\pi=: \xi_{0}<\xi_{1}<\xi_{2} \cdots<\xi_{K}:=\pi$. Set $R_{j}:=\left[\xi_{j}, \xi_{j+1}\right]$ for $j=0, \ldots, K-1$. As we pointed out in subsection 3.2, the decomposition $\mathcal{R}=\left\{R_{j}\right\}_{j=0}^{K-1}$ is compatible with $\mathcal{C}$, even though the points in $\mathcal{C}$ are at the endpoints of the intervals. In addition, $\mu\left(R_{j}\right)=\xi_{j+1}-\xi_{j}$ is just the length of the arc $R_{j}$. The mesh norm $\delta_{\mathcal{C}}$ and partition norm $\|\mathcal{R}\|$ are

$$
\begin{aligned}
\delta_{\mathcal{C}} & =\frac{1}{2} \max _{0 \leq j \leq K-1}\left|\xi_{j+1}-\xi_{j}\right|, \\
\|\mathcal{R}\| & =\max _{0 \leq j \leq K-1}\left|\xi_{j+1}-\xi_{j}\right| \leq 2 \delta_{\mathcal{C}} .
\end{aligned}
$$

The last equation above implies that if $\delta_{\mathcal{C}}<\eta /(2 n)$, then $\|\mathcal{R}\|<\eta / n$. Using Proposition 3.4 in place of Theorem 3.1 in the proof of Theorem 4.1 above yields the following result.

Corollary 4.1. For the case of the circle, if $0<\eta<\frac{1}{2}$ and $\delta_{\mathcal{C}}<\eta /(2 n)$, then there exist nonnegative numbers $w_{j}$ such that for every trigonometric polynomial $P \in \Pi_{n}^{1}$, we have

$$
\int_{-\pi}^{\pi} P(\theta) d \theta=\sum_{j=0}^{K-1} w_{j} P\left(\xi_{j}\right)
$$

where

$$
\left\|\left(\frac{w_{j}}{\xi_{j+1}-\xi_{j}}\right)\right\|_{\mathcal{C}, p^{\prime}} \leq(2 \pi)^{1 / p^{\prime}}(1-\eta)^{-1}, \quad 1 \leq p^{\prime}:=\frac{p}{p-1} \leq \infty .
$$

If one has $\frac{1}{2} \leq \eta<1$, then (4.8) and (4.9) continue to hold, but the coefficients $\left\{w_{j}\right\}$ may fail to be positive.

4.3. Computation of positive weights. The results established in Theorem 4.1 give the existence of positive weights, provided $\eta<\frac{1}{2}$ is satisfied; that is,

$$
\|\mathcal{R}\|<\frac{\eta}{n} \varrho_{q}<\frac{1}{2 n} \varrho_{q},
$$

where $\varrho_{q}$ is defined in (3.25). As we pointed out in the remark after Theorem 4.1 this condition is equivalent to one involving the cardinality of $\mathcal{C}$ and the dimension of the space we are integrating exactly. There are, however, two items that need to be addressed.

The constant $\varrho_{q}$ depends on a number of factors: the various constants used in connection with doubling weights; an estimate on the uniform bound on the norms of the Cesàro operators; and the constant relating norms of the delayed mean operators to the norms of the Cesàro operators. For general $q \geq 3$ these are not known. For $q=1$, all of the constants are determined. For the important case $q=2$, numerical experiments indicate that an estimate on the uniform bound for Cesàro operators involved (namely the $\Sigma_{n}^{(1)}$ 's) is 3 ; however, we know of no theoretical estimates. As we pointed out in subsection 2.2, the delayed mean operators $T_{n}^{(1)}$ have norms that are $3\left\|\Sigma_{n}^{(1)}\right\|$, giving a uniform bound for them of roughly 9 . However, for the $q=2$ case, the constants related to doubling weights are unknown. Thus, 
except for $q=1$, the constant $\varrho_{q}$ is unknown. This raises the question, how do we know that we have satisfied the required inequality? We will come back to this.

If we have satisfied the condition that $\eta<\frac{1}{2}$, then there exist positive weights for the quadrature formulas. How do we compute them?

Take $p=1$ in Theorem 4.1, and assume that (3.27) holds. The coefficients $a_{\xi}$ then satisfy the following constrained linear system:

$$
\left\{\begin{aligned}
0 & \leq a_{\xi} \leq \frac{\mu\left(R_{\xi}\right)}{1-\eta}, \xi \in \mathcal{C}, \\
\omega_{g} & =\sum_{\xi \in \mathcal{C}} a_{\xi}, \\
0 & =\sum_{\xi \in \mathcal{C}} a_{\xi} Y_{\ell, k}(\xi), k=1 \ldots d_{\ell}^{q}, \ell=1 \ldots n .
\end{aligned}\right.
$$

This system, which we know has at least one solution, will undoubtedly have many. In the problem of spherical design [8], one requires that the weights $a_{\xi}$ all be equal, which is possible only for a special set of points. From the middle equation in (4.10), we see that the average weight is $\bar{a}:=\left\langle\left(a_{\xi}\right)\right\rangle=\frac{\omega_{g}}{|\mathcal{C}|}$, and that the variance is given by

$$
\operatorname{var}(a):=\left\langle\left(a_{\xi}-\frac{\omega_{g}}{|\mathcal{C}|}\right)^{2}\right\rangle=\left\|\left(a_{\xi}\right)\right\|_{\ell^{2}}^{2}-\frac{\omega_{g}^{2}}{|\mathcal{C}|} .
$$

This shows that minimizing the variance - which amounts to choosing weights to be as close to the same as possible - is equivalent to minimizing the norm $\left\|\left(a_{\xi}\right)\right\|_{\ell^{2}}$. This leaves us with a feasible quadratic programming problem: minimize $\left\|\left(a_{\xi}\right)\right\|_{\ell^{2}}$, subject to the constrained linear system in (4.10). This always has a unique solution, and there are many standard algorithms that can be used to solve it.

Not only are there many algorithms, but there are fast algorithms for solving quadratic programming problems. This gives us a way of getting around knowing the condition that we mentioned above is satisfied. From dimensional arguments, start with $n \approx \omega_{q}^{1 / q} /\|\mathcal{R}\|$. If the program converges, then positive weights are obtained. If not, there are two choices. Lower $n$ or add sites that lower $\|\mathcal{R}\|$.

We close by remarking that we are currently working on getting good, practical bounds on the various constants mentioned above.

\section{REFERENCES}

1. L. Bos, N. Levenberg, P. Milman, and B. A. Taylor, Tangential Markov inequalities characterize algebraic submanifolds of $\mathbb{R}^{N}$, Ind. Univ. Math. J., 44 (1995), 115-138. MR 96i:41009

2. J. R. Driscoll and D. M. Healy, Computing Fourier Transforms and Convolutions for the 2-Sphere, Adv. in Appl. Math., 15 (1994), 202-250. MR 95h:65108

3. N. Dunford and J. T. Schwartz, Linear operators, Part I, Interscience, New York, 1958. MR 22:8302

4. T. Erdélyi, Notes on inequalities with doubling weights, J. Approx. Theory 100 (1999), 60-72. CMP 2000:01

5. H. Flanders, Differential Forms, Academic Press, New York, 1963. MR 28:5397

6. W. Freeden, O. Glockner, and M. Schreiner, Spherical Panel Clustering and Its Numerical Aspects, AGTM Report No. 183, University of Kaiserlautern, Geomathematics Group, 1997.

7. W. Freeden and U. Windheuser, Spherical Wavelet Transform and Its Discretization, AGTM Report No. 125, University of Kaiserlautern, Geomathematics Group, 1995.

8. J. M. Goethals and J. J. Seidel, Cubature formulae, polytopes, and spherical designs, in The Geometric Vein, Coexeter Festschrift, (C. Davis et al., eds.), Springer, New York, 1981, pp. 203-218. MR 83k:05033

9. R. B. Holmes, Geometric functional analysis and its applications, Springer-Verlag, New York, 1975. MR 53:14085

10. K. Jetter, J. Stöckler, and J. D. Ward, Error estimates for scattered data interpolation, Math. Comp., 68 (1999), 743-747. MR 99i:41032 
11. K. Jetter, J. Stöckler, and J. D. Ward, Norming sets and spherical cubature formulas, in Computational Mathematics, (Chen, Li, C. Micchelli, Y. Xu, eds.), Marcel Decker, New York, 1998, pp. 237-245. MR 99i:65022

12. G. Mastoianni and V. Totik, Weighted polynomial inequalities with doubling and $A_{\infty}$ weights, to appear in J. London Math. Soc.

13. H. N. Mhaskar, F. J. Narcowich and J. D. Ward, Approximation Properties of Zonal Function Networks Using Scattered Data on the Sphere, Adv. in Comp. Math., to appear.

14. H. N. Mhaskar and J. Prestin, Marcinkiewicz-Zygmund Inequalities, in Approximation Theory: In Memory of A. K. Varma, (N. K. Govil, R. N. Mohapatra, Z. Nashed, A. Sharma, and J. Szabados Eds.), Marcel Dekker, to appear.

15. C. Müller, Spherical Harmonics, Lecture Notes in Mathematics, Vol. 17, Springer Verlag, Berlin, 1966. MR 33:7593

16. P. Petrushev, Approximation by Ridge Functions and Neural Networks, SIAM J. Math. Anal., 30 (1999), 155-189. MR 99g:41031

17. D. Potts, G. Steidl, and M. Tasche, Fast algorithms for discrete polynomial transforms, Math. Comp., 67 (1998), 1577-1590. MR 99b:65183

18. E. M. Stein, Interpolation in polynomial classes and Markoff's inequality, Duke Math. J., 24 (1957), 467-476. MR 19:956b

19. E. M. Stein and G. Weiss, Introduction to Fourier analysis on Euclidean spaces, Princeton University Press, Princeton, New Jersey, 1971. MR 46:4102

20. G. Szegö, Orthogonal polynomials, Amer. Math. Soc. Colloq. Publ. 23, Amer. Math. Soc., Providence, 1975. MR 51:8724

21. A. Zygmund, A remark on conjugate series, Proc. London Math. Soc., 34 (1932), 392-400.

Department of Mathematics, California State University, Los Angeles, CA 90032

E-mail address: hmhaskar@calstatela.edu

Department of Mathematics, Texas A\&M University, College Station, TX 77843-3368

E-mail address: fnarc@math.tamu.edu

Department of Mathematics, Texas A\&M University, College Station, TX 77843-3368

E-mail address: jward@math.tamu.edu 